\title{
Effect of scapular stabilization exercise program in patients with subacromial impingement syndrome: a systematic review
}

\author{
Hariharasudhan Ravichandran 1,* Balamurugan Janakiraman², Asmare Yitayeh Gelaw², Berihu Fisseha', Subramanian Sundaram³, \\ Hidangmayum Richa Sharma ${ }^{4}$ \\ 'Department of Physiotherapy, School of Medicine, College of Health Sciences, Ayder Comprehensive Specialized Hospital, Mekelle University, Mekelle, Ethiopia \\ 2Department of Physiotherapy, School of Medicine, College of Medicine and Health Sciences, University of Gondar Comprehensive Specialized Hospital, University of \\ Gondar, Gondar, Ethiopia \\ ${ }^{3}$ Department of Physiotherapy, Sree Balaji College of Physiotherapy, Chennai, India \\ ${ }^{4}$ Department of Physiotherapy, Sakra Institute of Rehabilitation Sciences, Sakra World Hospital, Bangalore, India
}

Scapular stabilization approaches have been a mainstay of therapeutic training programs in the recent past for patients with subacromial impingement syndrome, whereas its contributions solely in the clinical outcome of reducing shoulder pain and disability are largely unclear. This systematic review aims to evaluate and summarize the best evidence regarding the role of scapular stabilization interventions in alleviating shoulder dysfunction among subjects with subacromial impingement syndrome. Six reviewers involved in this systematic review. Literature was retrieved systematically through searching 5 electronic databases (PubMed, MEDLINE, CINAHL, Cochrane, and Google Scholar). Articles published from the year 2010 up to and including 2019 were included. The literature search included clinical trials those intervened subjects with scapular exercises or scapular stabilization exercises or scapular rehabilitation, as an intervention for subacromial impingement syndrome. Seven studies, totaling 228 participants were included in this systematic review. Articles included in this review were graded ac- cording to Lloyd-Smith hierarchy of evidence scale and critically appraised with a tool developed by National, Heart, Lung and Blood Institute (United States), named as quality assessment of controlled intervention studies tool. There was a significant effect on the scapular stabilization exercise program on improving pain and disability among subjects with subacromial impingement syndrome. This systematic review provides sufficient evidence to suggest that scapular stabilization exercises offers effectiveness in reducing pain and disability among subjects with subacromial impingement syndrome. However, more trials with larger sample are needed to provide a more definitive evidence on the clinical outcomes of scapular stabilization exercises among patients with impingement.

Keywords: Exercise, Shoulder impingement syndrome, Subacromial impingement syndrome, Scapular stabilization, Scapular exercises, Shoulder rehabilitation

\section{INTRODUCTION}

Globally more than 1.7 billion people are estimated to be affected by musculoskeletal disorders. Musculoskeletal disorders are often associated with long term pain, functional limitation, disability, productivity loss, and poor quality of life (Fatoye, 2018). Shoulder pain is one of the (GBD 2016 Disease and Injury Inci- dence and Prevalence Collaborators, 2017) common presentations with the prevalence ranging between $7 \%$ and $26 \%$ (Luime et al., 2004). Globally, about $4 \%$ of adults visit their general practitioner for shoulder pain It is estimated that approximately 1.5 million shoulder pain consultations in England account for an estimated annual cost of $£ 100$ million (Greving et al., 2012). The mean annual cost of shoulder conditions per patient has been estimated at

\footnotetext{
${ }^{*}$ Corresponding author: Hariharasudhan Ravichandran

(iD) https://orcid.org/0000-0002-0703-9675

Department of Physiotherapy, School of Medicine, College of Health Sciences, Ayder Comprehensive Specialized Hospital, Mekelle University, P.O. Box: 1871, Mekelle, Ethiopia

E-mail: hrkums63@gmail.com

Received: April 2, 2020 / Accepted: April 29, 2020
} tribution Non-Commercial License (https://creativecommons.org/licenses/by-nc/4.0/) which permits unrestricted non-commercial use, distribution, and reproduction in any medium, provided the original work is properly cited. 
$£ 3500$ in Sweden (Virta et al., 2012) and about $£ 8500$ in the United States, with the cost of surgical interventions and absence from work contribution to this estimates (Oh et al., 2007). Just about half of the subjects presenting with shoulder pain are related to subacromial impingement syndrome and the overall prognosis is highly variable with nearly $40 \%$ of the subjects reporting pain one year after consultation (van der Windt et al., 2019). Subacromial impingement syndrome is a multifactorial condition where the subacromial space, the area directly below the acromion process and above the shoulder joint, has narrowed (Larsson et al., 2019). Houglum identified pathological posture, overuse or repetitive trauma, age-related changes, poor vascularity, and fatigue of the scapular and glenohumeral muscles, biomechanical alterations, neuromuscular adaptations, and rotator cuff musculature imbalances as a root cause for this subacromial impingement syndrome (Houglum, 2013). Besides, variations in anatomical structures like acromion shape, subacromial osteoarthritic spurs, and poor scapular setting can present as internal or external impingement (Seitz et al., 2011).

There is growing evidence in support of exercises, to restore shoulder kinematics are frequently suggested as a treatment option for subjects presenting with subacromial impingement syndrome and sometimes surgical decompression, acromioplasty are employed to restore subacromial space in worst cases. Therapeutic exercises are reported to be effective in restoring shoulder function; these exercises are a combination of scapular stabilization exercises, rotator cuff strengthening exercises, range of motion (ROM), proprioceptive neuromuscular facilitation (PNF), and stretching exercises. Though several individual studies (Başkurt et al., 2011; Moezy et al., 2014; Struyf et al., 2013; Turgut et al., 2017) report the efficacy of scapular stabilization exercise in the rehabilitation of subacromial impingement syndrome, there is however lack of consensus of evidence as to which specific exercise technique(s) are effective clinically. Physiotherapy is the mainstay management for several musculoskeletal disorders, the cost of physiotherapy services for people with musculoskeletal disorders is raising, and it is high time that clinicians practice cost and clinically effective intervention techniques (Fatoye, 2018). Recent literature has recommended the need to address specific exercise strategies or programs that are aimed to improve scapular kinematics, muscle activity, strength, flexibility, and shoulder function in the rehabilitation of subacromial impingement syndrome. A perspective report and an algorithm guideline on the rehabilitation of shoulder supported scapular-focused treatment in patients with subacromial pain syndrome for better clinical outcome, at the same time re- ported that the current evidence for scapular-focused approaches are ambiguous and recommended more clinical metrics (McQuade et al., 2016). Scapular stabilization exercises are thought to have an important role in improving pain and dysfunction in the shoulder. The concept that an unstable scapula is associated with pathology and dysfunction of the shoulder is well accepted. The predominant theory emphasizes that for optimal function of the glenohumeral joint, the scapula must provide a stable base upon which upper extremity tasks are completed. Impaired scapular stability increases the risk for pathologies such as impingement or rotator cuff tears (McQuade et al., 2016). Improper movement of the scapula is often forgotten cause of pain and dysfunction (Panagiotopoulos and Crowther, 2019). Though alteration in scapular kinematics in people with impingement is evident, yet the prevalence and type of alterations vary substantially (Takeno et al., 2019). The majority of previously published literature reports a decreased scapular external rotation, decreased scapular upward rotation, and a decreased posterior scapular tilting in patients with subacromial impingement syndrome (Struyf et al., 2011). Scapular stabilization exercise aims to restore scapular position, orientations, motor control of muscles, and movement pattern, thereby attaining stability of scapula for better kinematics of shoulder. It is frequently prescribed as a main component of rehabilitation programs for different types of shoulder pain syndromes.

The significance of scapular stabilization approaches has drawn considerable attention from research scientists, educators, and clinicians. Several systematic reviews have focused on investigating the efficacy of a variety of physical therapy interventions and scapular-focused interventions for subjects with subacromial impingement syndrome (Desmeules et al., 2003; Green et al., 2003; Hanratty et al., 2012; Kromer et al., 2009). However, to the best of our knowledge, there has been no systematic review available in the literature on the clinical effects of a scapular stabilization approach in patients with subacromial impingement syndrome. Given the lack of consensus on the effectiveness of scapular stabilization exercises in improving shoulder function among subjects with subacromial impingement syndrome, there is a clear need for the agreement of evidence related to the efficacy of scapular stabilization exercises in treating subacromial impingement syndrome. The purpose of this systematic review was to evaluate the evidence of the effectiveness of scapular stabilization exercises on reducing pain and disability among subjects affected with subacromial impingement syndrome. Also, the key components impacting the clinical outcome and intervention will be identified. The conclusions drawn from this review should aid in the development of 
better understanding of the inclusion of scapular stabilization exercises as a part of clinical practice recommendations for the intervention of subacromial impingement syndrome.

\section{MATERIALS AND METHODS}

\section{Data sources}

This review was conducted as per the guideline of Preferred Reporting Items for Systematic Reviews and Meta-Analysis (PRISMA) statement (Moher et al., 2015). Six authors involved in this systematic review. Four review authors (HR, BJ, BF, and AY) independently conducted a search strategy. Articles were selected from the following electronic databases; PubMed, Cochrane Library, MEDLINE, Cumulative Index to Nursing and Allied Health Literature, and Google Scholar using medical subject headings and free text related to subacromial impingement syndrome ("impingement syndrome," "impingement," "shoulder impingement syndrome") and scapular stabilization exercises ("Scapular stabilization exercises," "Scapular exercises," "Scapular rehabilitation"). Articles published from 2010 and up to 2019 were searched. Suggested keywords used for the search strategy were illustrated in Table 1.

\section{Study selection}

Two reviewers (SS and RS) independently performed the study selection process. Randomized controlled trials, nonrandomized clinical trials, and quasi-experimental study design studies inter-

Table 1. Keywords and syntax used in search strategy

\begin{tabular}{|c|c|c|c|c|c|c|}
\hline No. & Keywords & Pubmed & MEDLINE & CINAHL & Cochrane & $\begin{array}{l}\text { Google } \\
\text { Scholar }\end{array}$ \\
\hline 1 & Impingement & 25,673 & 723 & 3,891 & 1,279 & 39,742 \\
\hline 2 & $\begin{array}{l}\text { Shoulder impingement } \\
\text { syndrome }\end{array}$ & 2,878 & 219 & 210 & 685 & 5,561 \\
\hline 3 & $\begin{array}{l}\text { Subacromial } \\
\text { impingement }\end{array}$ & 2,109 & 187 & 128 & 541 & 6,723 \\
\hline 4 & Above 1-3 & 1,578 & 521 & 4,101 & 2,276 & 49,888 \\
\hline 5 & Scapular stabilization & 239 & 13 & 328 & 187 & 2,321 \\
\hline 6 & Scapular exercises & 288 & 21 & 23 & 256 & 1,287 \\
\hline 7 & Scapular rehabilitation & 451 & 122 & 38 & 478 & 3,456 \\
\hline 8 & Above 5-7 & 326 & 118 & 381 & 734 & 6,733 \\
\hline 9 & $\begin{array}{l}\text { Randomized controlled } \\
\text { trial }\end{array}$ & 2,233 & 424 & 129 & 2,109 & 5,925 \\
\hline 10 & $\begin{array}{l}\text { Experimental study } \\
\text { design }\end{array}$ & 938 & 232 & 210 & 167 & 4,390 \\
\hline 11 & Above 9-10 & 3,054 & 654 & 318 & 2,189 & 9,872 \\
\hline 12 & Above 4+8+11 & 854 & 25 & 63 & 49 & 1,298 \\
\hline
\end{tabular}

vening scapular stabilization exercises or approaches in adult subjects diagnosed with subacromial impingement syndrome were considered for selection.

\section{Criteria for inclusion}

To ascertain that all relevant studies were selected, two reviewers (BJ and $\mathrm{BF}$ ) screened the included papers with the following inclusion criteria: (a) Original research reports of data collected on the subjects presenting or diagnosed with subacromial impingement syndrome; (b) studies evaluating pain or disability or movement as an outcome; (c) availability of full-text; (d) studies published in English and other languages with an English version.

\section{Hierarchy of evidence}

Two reviewers (HR and AY) assessed the level of evidence among included papers using the Hierarchical system of Lloyd-Smith (Lloyd-Smith, 1997). Articles with a high level of evidence ranging between 1a and $2 \mathrm{~b}$ on the Lloyd-Smith scale were included in this review (Table 2).

\section{Data extraction}

Three reviewers (HR, BJ, and SS) independently extracted data using a structured data extraction form adapted from the Cochrane collaboration (Cochrane Effective Practice and Organisation of Care, 2013). Informations that were extracted include the following items: general study information (title of the article, year of publication, first author's last name, and country of study); study design and characteristics (participant characteristics, potential predictors, and outcome measure); and findings including the length of follow-up. Any disagreements or conflicts during and after data

Table 2. Lloyd-Smith hierarchy of evidence

\begin{tabular}{|c|c|c|}
\hline $\begin{array}{l}\text { Level of } \\
\text { evidence }\end{array}$ & Study design & Articles \\
\hline 1a & $\begin{array}{l}\text { Meta-analysis of randomized controlled } \\
\text { study }\end{array}$ & \\
\hline $1 b$ & Individual randomized controlled study & $\begin{array}{l}\text { Baskurt et al. (2011) } \\
\text { Struyf et al. (2013) } \\
\text { Moezy et al. (2014) } \\
\text { Turgut et al. (2017) }\end{array}$ \\
\hline $2 a$ & $\begin{array}{l}\text { Well designed, non randomized } \\
\text { controlled study }\end{array}$ & Hotta et al. (2017) \\
\hline $2 b$ & Well designed quasi experimental study & $\begin{array}{l}\text { Bernardsson et al. (2011) } \\
\text { Shankar et al. (2016) }\end{array}$ \\
\hline 3 & $\begin{array}{l}\text { Nonexperimental descriptive } \\
\text { studies- comparative/case studies }\end{array}$ & \\
\hline 4 & Respectable opinion & \\
\hline
\end{tabular}


extraction was resolved by a mutual agreement, and if needed, by requesting the opinion of the third reviewer (AY). Computerized and head searching resulted in 2,292 potentially appropriate articles, with 7 articles meeting the inclusion criteria of this review.

\section{Quality appraisal}

The quality of the included full-text articles was evaluated by three reviewers (BJ, AY, and RS) based on a tool developed by the National Heart, Lung and Blood Institute (United States), named as quality assessment of controlled intervention studies. (National Heart, Lung and Blood Institute, 2014). The quality of each paper was scored according to the items listed in the quality assessment of controlled intervention studies tool. The lowest possible score was 0 , and the maximum possible score was 14 . Based on

Table 3. Quality assessment of included articles

\begin{tabular}{|c|c|c|c|c|c|c|c|}
\hline Criteria & $\begin{array}{l}\text { Baskurt et al. } \\
\text { (2011) }\end{array}$ & $\begin{array}{l}\text { Bernardsson et } \\
\text { al. (2011) }\end{array}$ & $\begin{array}{l}\text { Struyf et al. } \\
\text { (2013) }\end{array}$ & $\begin{array}{l}\text { Moezy et al. } \\
\text { (2014) }\end{array}$ & $\begin{array}{c}\text { Shankar et al. } \\
\text { (2016) }\end{array}$ & $\begin{array}{l}\text { Turgut et al. } \\
\text { (2017) }\end{array}$ & $\begin{array}{l}\text { Hotta et al. } \\
\text { (2017) }\end{array}$ \\
\hline $\begin{array}{l}\text { 1. Was the study described as randomized, a } \\
\text { randomized trial, a randomized clinical trial, } \\
\text { or an RCT? }\end{array}$ & Yes & No & Yes & Yes & No & Yes & No \\
\hline $\begin{array}{l}\text { 2. Was the method of randomization } \\
\text { adequate (i.e., use of randomly generated } \\
\text { assignment)? }\end{array}$ & Yes & No & Yes & Yes & No & Yes & No \\
\hline $\begin{array}{l}\text { 3. Was the treatment allocation concealed (so } \\
\text { that assignments could not be predicted)? }\end{array}$ & Not mentioned & No & Yes & Yes & No & Not mentioned & No \\
\hline $\begin{array}{l}\text { 4. Were study participants and providers } \\
\text { blinded to treatment group assignment? }\end{array}$ & No & Not mentioned & Yes & Yes & Not mentioned & Not mentioned & No \\
\hline $\begin{array}{l}\text { 5. Were the people assessing the outcomes } \\
\text { blinded to the participants' group } \\
\text { assignments? }\end{array}$ & No & Not mentioned & Yes & Yes & Not mentioned & Not mentioned & No \\
\hline $\begin{array}{l}\text { 6. Were the groups similar at baseline on } \\
\text { important characteristics that could affect } \\
\text { outcomes (e.g., demographics, risk factors, } \\
\text { co-morbid conditions)? }\end{array}$ & Yes & Yes & Yes & Yes & Yes & Yes & Yes \\
\hline $\begin{array}{l}\text { 7. Was the overall drop-out rate from the } \\
\text { study at endpoint } 20 \% \text { or lower of the } \\
\text { number allocated to treatment? }\end{array}$ & Yes & Yes & Yes & Yes & Yes & Yes & Yes \\
\hline $\begin{array}{l}\text { 8. Was the differential drop-out rate (between } \\
\text { treatment groups) at endpoint 15\% points } \\
\text { or lower? }\end{array}$ & Yes & Yes & Yes & Yes & Yes & Yes & Yes \\
\hline $\begin{array}{l}\text { 9. Was there high adherence to the intervention } \\
\text { protocols for each treatment group? }\end{array}$ & Yes & Yes & Yes & Yes & Yes & Yes & Yes \\
\hline $\begin{array}{l}\text { 10. Were other interventions avoided or } \\
\text { similar in the groups (e.g., similar } \\
\text { background treatments)? }\end{array}$ & Yes & Yes & Yes & Yes & Yes & Yes & Yes \\
\hline $\begin{array}{l}\text { 11. Were outcomes assessed using valid and } \\
\text { reliable measures, implemented } \\
\text { consistently across all study participants? }\end{array}$ & Yes & Yes & Yes & Yes & Yes & Yes & Yes \\
\hline $\begin{array}{l}\text { 12. Did the authors report that the sample size } \\
\text { was sufficiently large to be able to detect a } \\
\text { difference in the main outcome between } \\
\text { groups with at least } 80 \% \text { power? }\end{array}$ & Not mentioned & Yes & Yes & Yes & Yes & Yes & Yes \\
\hline $\begin{array}{l}\text { 13. Were outcomes reported or subgroups } \\
\text { analyzed prespecified (i.e., identified before } \\
\text { analyses were conducted)? }\end{array}$ & Yes & Yes & Yes & Yes & Yes & Yes & Yes \\
\hline $\begin{array}{l}\text { 14. Were all randomized participants analyzed } \\
\text { in the group to which they were originally } \\
\text { assigned, i.e., did they use an } \\
\text { intention-to-treat analysis? }\end{array}$ & Not mentioned & Not mentioned & Not mentioned & Not mentioned & Not mentioned & Not mentioned & Not mentioned \\
\hline Scores & $9 / 14$ & $8 / 14$ & $10 / 14$ & $13 / 14$ & $8 / 14$ & $10 / 14$ & $8 / 14$ \\
\hline
\end{tabular}

$\mathrm{RCT}$, randomized controlled trials. 
Table 4. Risk of bias in included studies

\begin{tabular}{|c|c|c|c|c|c|c|c|}
\hline Bias & $\begin{array}{l}\text { Baskurt et al. } \\
\text { (2011) }\end{array}$ & $\begin{array}{l}\text { Bernardsson et al. } \\
\text { (2011) }\end{array}$ & $\begin{array}{l}\text { Sturyf et al. } \\
\text { (2013) }\end{array}$ & $\begin{array}{l}\text { Moezy et al. } \\
\text { (2014) }\end{array}$ & $\begin{array}{l}\text { Shankar et al. } \\
\text { (2016) }\end{array}$ & $\begin{array}{l}\text { Turgut et al. } \\
\text { (2017) }\end{array}$ & $\begin{array}{l}\text { Hotta et al. } \\
\text { (2017) }\end{array}$ \\
\hline Selection of participants & Low risk & Low risk & Low risk & Low risk & Low risk & Low risk & High risk \\
\hline Allocation concealment & Low risk & High risk & Low risk & Low risk & High risk & Low risk & High risk \\
\hline Blinding of participants & Unclear & Unclear & Unclear & Low risk & High risk & Unclear & Unclear \\
\hline Assessor blinding & Unclear & Low risk & Low risk & Low risk & High risk & Unclear & Unclear \\
\hline Incomplete data & Low risk & Low risk & Low risk & Low risk & Low risk & Low risk & Low risk \\
\hline Selective reporting & Low risk & Low risk & Low risk & Low risk & Low risk & Low risk & Low risk \\
\hline Other Bias & Unclear & Unclear & Unclear & Unclear & Unclear & Unclear & Low risk \\
\hline
\end{tabular}

the methodological quality, this review categorized each study as low $(0-4)$, moderate $(5-10)$, or high (11-14). The consistency of scoring and categorizing across the reviewers was ensured before proceeding further. Disagreements among the reviewers were solved by consensus building. In this review, 5 articles were graded as moderate quality and 2 articles were graded as high quality respectively (Table 3).

\section{Risk of bias in the included studies}

Two reviewers (SS and BF) independently assessed the risk of bias in the included trials using the Cochrane Collaboration tool for assessing risk of bias (Sterne et al., 2019). The items that were assessed for potential bias by the tool include; sequence generation, allocation concealment, blinding of participants and personnel, blinding of outcome assessment, incomplete outcome data, selective reporting, and other bias (Table 4).

\section{RESULTS}

\section{Study search}

A total of 7 articles were identified for inclusion in this review. The PRISMA flow diagram (Moher et al., 2015) in Fig. 1 illustrates the selection process.

\section{Study characteristics}

The 7 trials analyzed in this review included a total of 228 participants, with 119 allocated to scapular stabilization exercise intervention and 109 to control groups. The length of follow-up varied from 2 to 12 weeks (Table 5). The mean quality score of the included trials was 9.42 out of a total of 14 possible points. The interrater agreement calculated based on the kappa statistics was 0.884 .

\section{Risk of bias in included studies}

Random sequence generation was reported in 6 articles and not done in Hotta et al. (2018). Allocation concealment had high risk

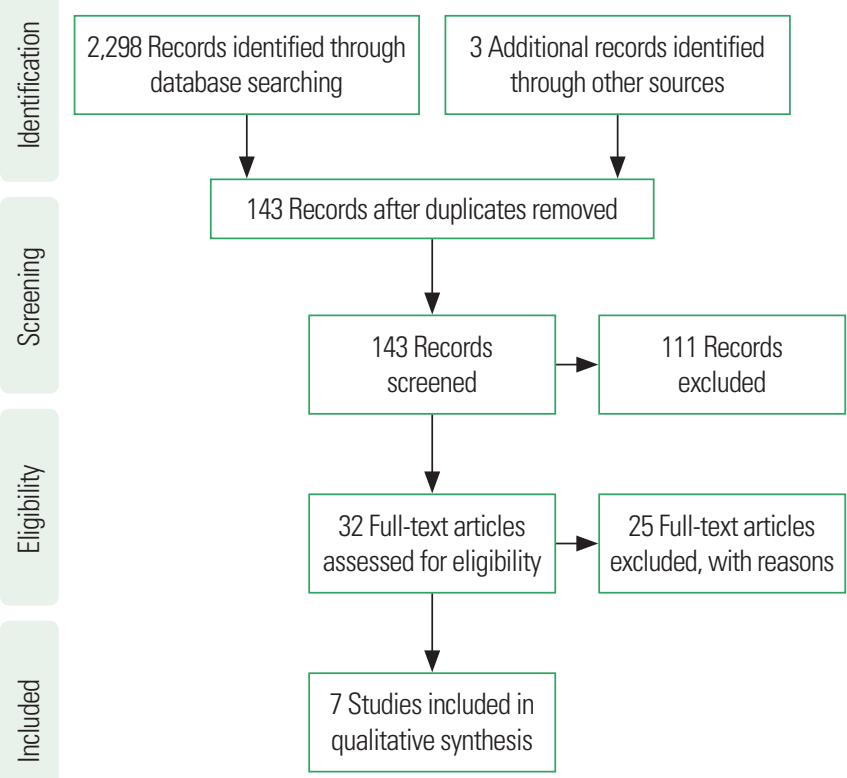

Fig. 1. PRISMA (Preferred Reporting Items for Systematic Reviews and Meta-Analyses) flow diagram illustrating the selection process.

in 3 articles (Bernhardsson et al., 2011; Hotta et al., 2018; Shankar et al., 2016). Blinding of participants to study group allocation was achieved in one study, not achieved in one, and not reported in five studies. Trial personnel or outcome assessors were not blinded to allocation in one article (Shankar et al., 2016) and not reported in 3 articles. Incomplete outcome data and selective reporting had a complete low-risk percentage. In summary, the study by Struyf et al. (2013) was determined to have low risk of bias and two articles, by Hotta et al. (2018) and Shankar et al. (2016) were judged to have a high risk of bias (Figs. 2, 3). The interrater agreement calculated based on the kappa statistics was 0.901.

\section{Result of individual studies}

Başkurt et al. (2011) investigated the effectiveness of the scapu- 


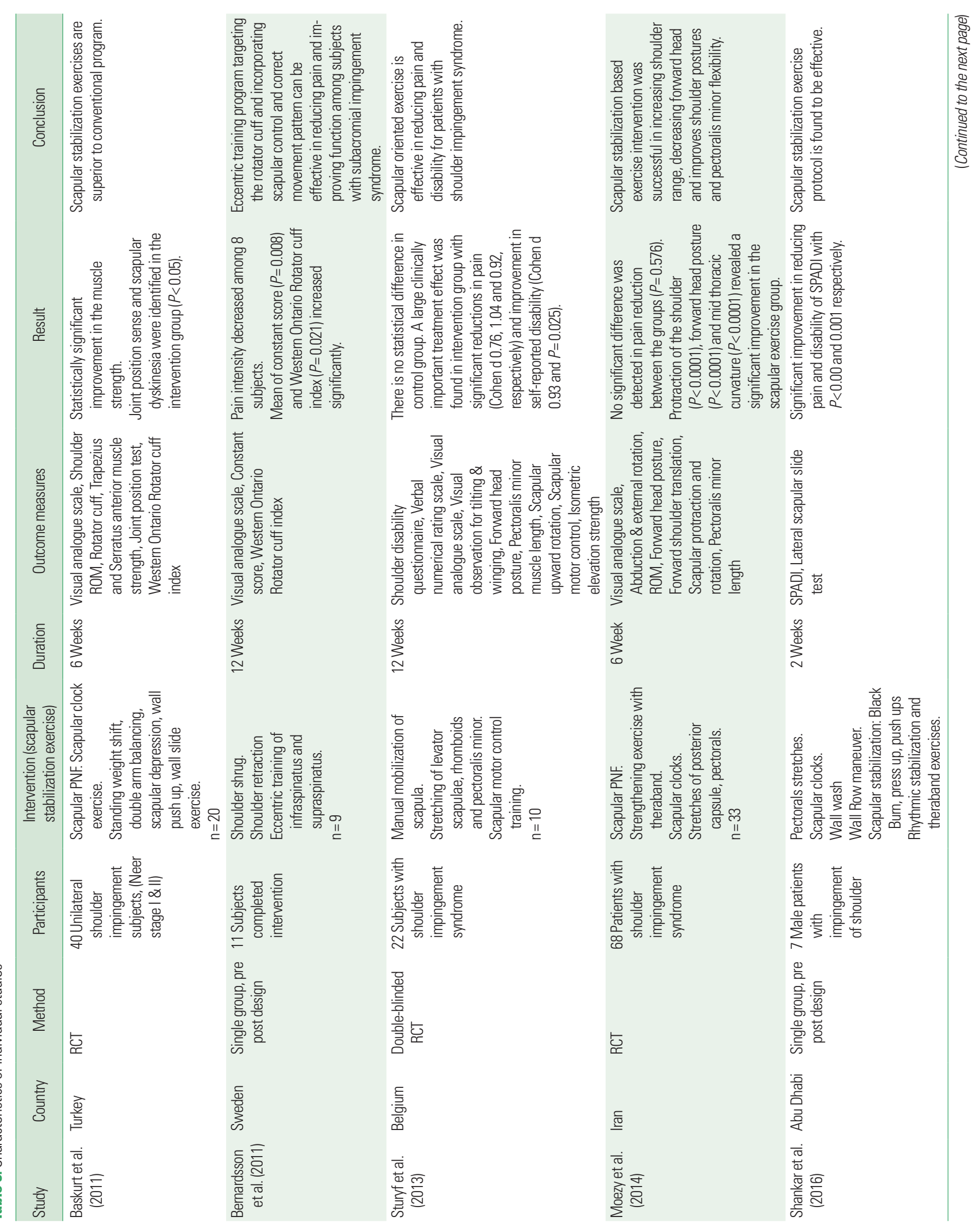




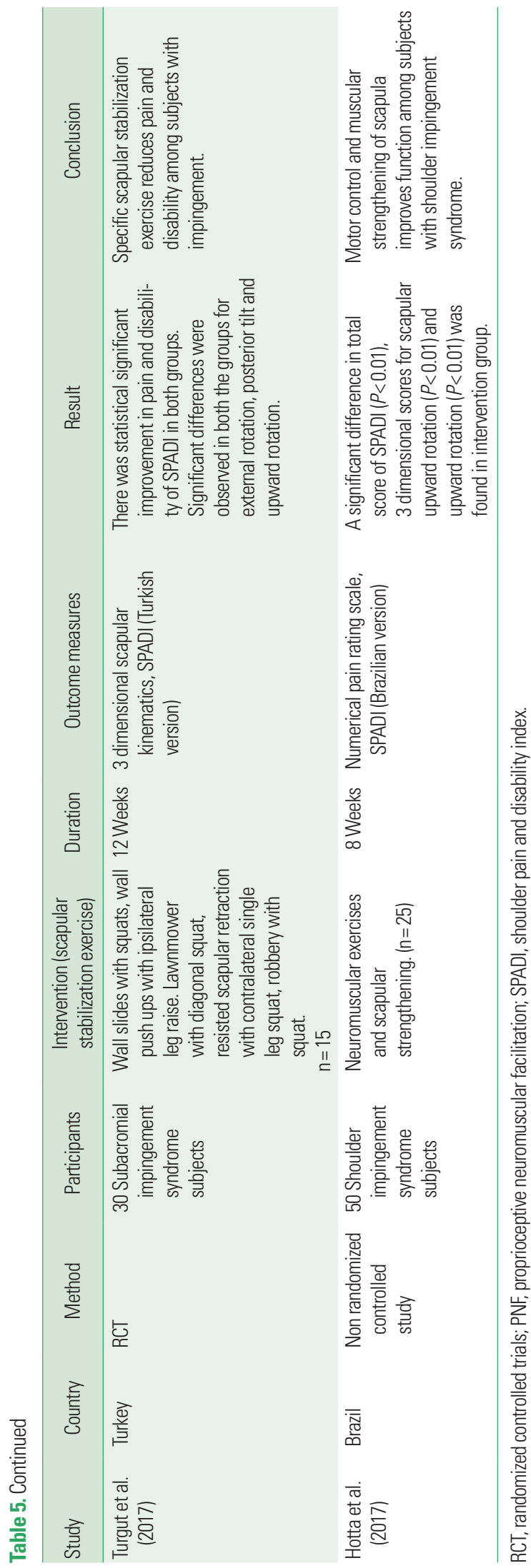

lar stabilization approach among 40 patients with subacromial impingement syndrome in Turkey. In this randomized controlled trial, the scapular stabilization group $(\mathrm{n}=20)$ was compared with the stretching and strengthening group $(\mathrm{n}=20)$ for 6 weeks. Outcome measures were pain intensity, shoulder ROM, scapular muscle strength, joint position test, Western Ontario Rotator cuff index. Both groups achieved statistical improvement in all the outcomes, meanwhile more significant differences were found in the scapular stabilization exercise group in improving scapular muscle strength and in joint position tests. It was concluded that scapular stabilization exercise is more superior to the conventional program among subjects with subacromial impingement syndrome.

Bernhardsson et al. (2011) evaluated the effect of 12-week eccentric based scapular exercise among 9 patients with subacromial impingement syndrome. This was a single-subject experimental design conducted in Sweden. After baseline measures of visual analogue scale, constant score, and Western Ontario Rotator cuff index, subjects underwent interventions of the eccentric infraspinatus and supraspinatus exercise, shoulder shrugs, and retraction, stretching of the trapezius. Post interventional outcomes showed that there was a significant improvement in visual analogue scale in reducing pain with improvement in function demonstrated by constant score $(P=0.008)$ and Western Ontario Rotator cuff index $(P=0.021)$. Therefore Scapular control can be effective in decreasing pain and improving function among subjects with subacromial impingement syndrome.

Struyf et al. (2013) compared the effectiveness of scapular-focused treatment with control therapy in Belgium. Following baseline measures, 22 subjects were randomized into the scapular orientation group and control group and treated for 12 weeks. Post interventional between-group measures of self-reported questionnaire $(P=0.025)$, verbal numerical rating scale $(P=0.022)$, visual analogue scale $(P=0.046)$ favored the intervention group. Those authors stated that scapular oriented exercise is effective in reducing pain and disability in patients with shoulder impingement syndrome.

Moezy et al. (2014) conducted a randomized controlled trial in Iran to compare the effectiveness of scapular stabilization based exercise with conventional physical therapy in 68 patients with shoulder impingement syndrome. After 6 weeks, there was significant improvement in shoulder abduction $(P=0.024)$ and external rotation $\operatorname{ROM}(P=0.001)$, improvement in forward shoulder translation $(P<0.0001)$ and flexibility $(P<0.0001)$ between the groups. Protraction of shoulder $(P=0.001)$, forward head posture $(P=0.001)$ and thoracic curvature $(P=0.001)$ revealed a significant improve- 


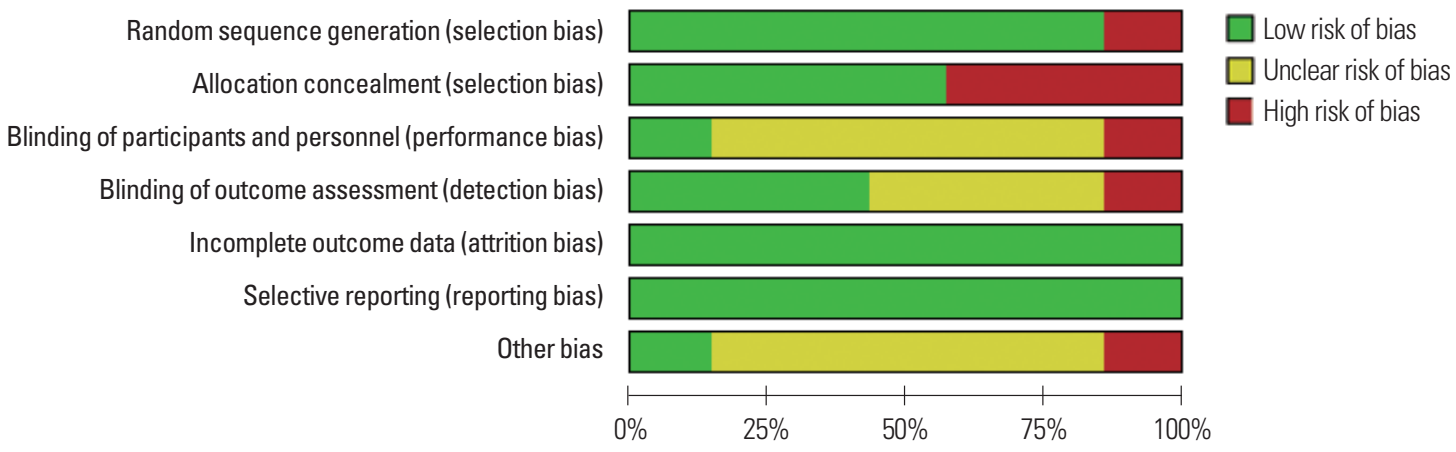

Fig. 2. Risk of bias graph: author's judgment about each risk of bias item presented as percentages across all included studies.

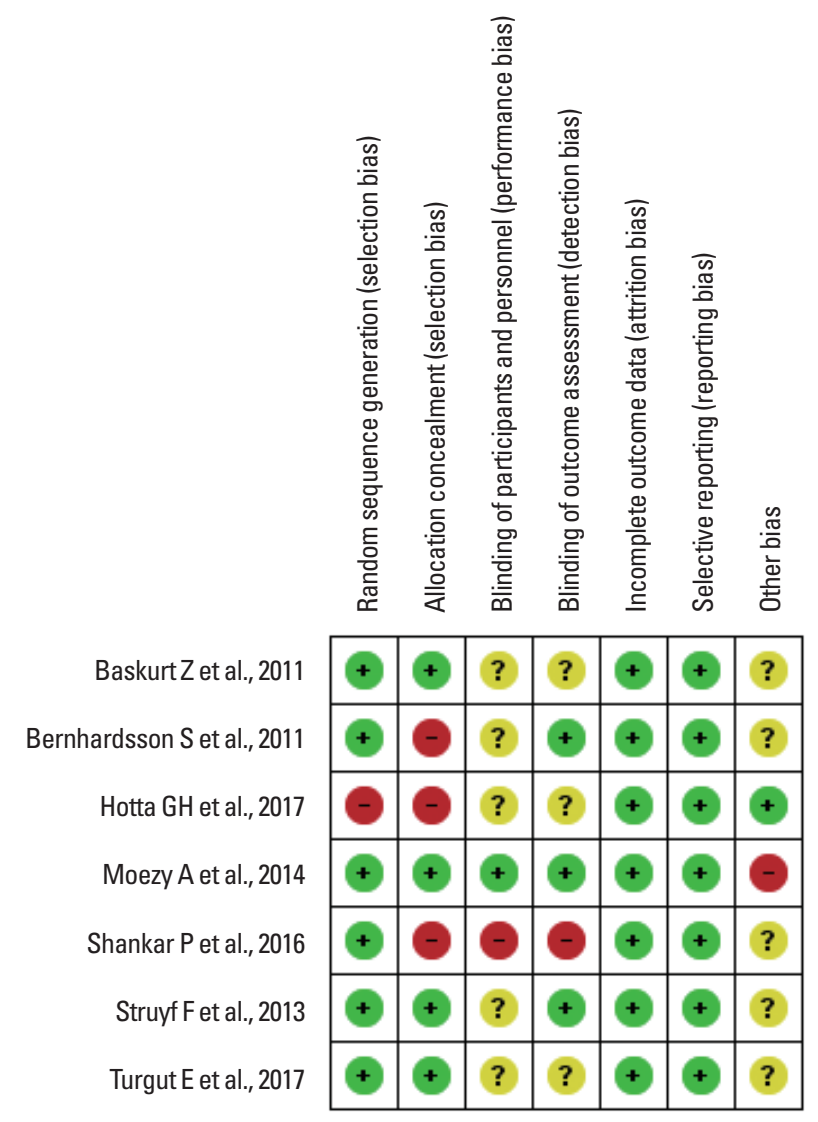

Fig. 3. Risk of bias assessment of included trials.

ment in the scapular stabilization group. No significant differences were detected in pain reduction $(P=0.576)$. This concludes that scapular stabilization exercises are effective in increasing ROM of shoulder and pectorals flexibility, decreasing forward head and poor shoulder posture.

Shankar et al. (2016), performed a single group pre-post study among 7 shoulder impingement subjects in Iran. Scapular stabili- zation program was implemented for 2-week duration. Paired $t$-test analysis found that there is a statistically significant difference $P<0.000$ in shoulder pain and disability index and lateral scapular slide test. The study also concluded that scapular stabilization exercise protocol is effective in improving pain and disability among shoulder impingement subjects.

Turgut et al. (2017), investigated the effect of scapular stabilization exercise $(n=15)$ with conventional exercises $(n=15)$ in Turkey. The study was conducted for 12 weeks. Both the groups demonstrated improvement in 3-dimensional scapular kinematics for external rotation, posterior tilt and upward rotation of the scapula and shoulder pain and disability index scale for pain and disability. Statistical values are higher in the intervention group when compared with the control group. It is proved that specific scapular stabilization exercises decrease pain and disability in impingement syndrome.

Hotta et al. (2018) in Brazil analyzed the effect of scapula focused exercise protocol in impingement syndrome. Fifty subjects were randomized into both intervention $(\mathrm{n}=25)$ and control group $(n=25)$, interventions were given for 8 weeks duration. Scapular kinematics and shoulder pain and disability index were the outcome measures used. There was significant improvement in shoulder pain and disability index $(P<0.01)$ and Shoulder kinematics for upward rotation $(P<0.01)$, anterior tilt $(P<0.01)$ and internal rotation $(P<0.01)$ of scapula. These results indicate exercises focused on scapula are beneficial in alleviating impingement syndrome.

\section{Synthesis of results}

Principle findings of this systematic review are reported by LloydSmith hierarchy of evidence, quality assessment of articles, characteristics of included articles and risk of bias. Seven articles synthesized in this review demonstrated a varying statistical outcome. 
This systematic review included articles that provided comprehensive information from various parts of the world concerning the effectiveness of scapular stabilization exercise program among subacromial impingement syndrome subjects. All the authors conclude that scapular stabilization is effective, by comparing with conventional physiotherapy or control group. Two articles used a quasi-experimental single group pre-post design and concluded that the scapular stabilization exercise program is effective.

\section{DISCUSSION}

This systematic review investigated the evidence for the efficacy of scapular stabilization exercise for patients with subacromial impingement syndrome on reducing shoulder pain and disability among adult people. No previous systematic reviews reporting solely on the efficacy of scapular stabilization exercises on subacromial impingement syndrome was found. Therefore, to the best of our knowledge, this is the first systematic review to solely focus on the consensus of data concerning the effects of scapular stabilization exercise among adult subjects with subacromial impingement syndrome.

From the result of this review, it is hypothesized that scapular exercises are effective in reducing pain and disability among subjects with subacromial impingement syndrome. The number of trials available was limited, and the quality of these trials was moderate. The outcome measures were not consistent between trials for the measures of pain and disability, therefore preventing the pooling of results for a meta-analysis.

Seven articles including a total of 228 participants reported on more than 10 outcome measures (visual analogue scale, shoulder ROM, muscle power, Western Ontario rotator cuff index, constant score, joint position test, muscle flexibility, posture of neck \& shoulder, shoulder pain and disability index, 3-dimensional scapular movement test, etc.) which aimed to measure pain and disability among subjects with subacromial impingement syndrome.

Pain

To assess the intensity of pain, visual analogue scale was almost used by all the articles included in this review. The statistically significant changes in pain intensity can be considered clinically relevant. Strengthening of scapular stabilizers, stabilize the humeral head in the glenoid fossa while retaining the space between greater tubercle and acromion, thereby preventing any possible compression of structures in the space. A previous systematic review by Bury et al. (2016) among rotator cuff injury subjects in- dicated support in favor of scapular-focused exercise approaches, though the evidence was either conflicting or below clinical significance for variables such as pain.

\section{Disability}

Shoulder pain and disability index, Western Ontario rotator cuff index, constant score, muscle strength, and joint ROM demonstrated significant improvement statistically in the included articles. Scapular stabilization exercises improve muscle strength, motor control, movement pattern, joint position sense, and reduce disability in terms of shoulder pain and disability index and Western Ontario rotator cuff index. Increase in muscle strength, joint position sense, and repositioning of scapula restored normal scapula-humeral rhythm and hence reduces disability. Dewhurst (2010) found that impaired function of pectorals and levator scapulae muscles can affect the subacromial space negatively, thereby increasing the incidence of subacromial impingement syndrome. The author also proposed that to ensure biomechanically correct movement control of supraspinatus, infraspinatus, teres minor, and subscapularis these muscle group has to be strengthened.

The findings of this review are similar to the work of Schmitt and Snyder-Mackler (1999), which reported that scapula-thoracic weakness as a cause of secondary subacromial impingement syndrome. Hence, this review suggests that the scapular stabilization exercise program shall be used as an integral part of therapeutic intervention programs aiming at reducing pain and disability among subjects with subacromial impingement syndrome.

\section{Strength and limitations}

The current review provides shoulder rehabilitation specialists with insight into scapular stabilization exercises in improving pain for subacromial impingement subjects. This systematic review emphasizes the importance of focusing on scapular stabilization approaches in impingement syndrome, which is the basis for assessment and management of shoulder dysfunction secondary to impingement.

However, for benefits of future researches and interpretation of these findings with caution, the limitations to be considered are; the heterogeneity in terms of outcome measures of the reviewed studies, conducting a meta-analysis was not meaningful and the inclusion of studies published in English language or English version, which might have resulted in the exclusion of important findings. Further, the articles included were unclear about the stage or severity of impingement syndrome. 


\section{CONCLUSIONS}

Articles included in this review demonstrated effects supporting the use of scapular exercise as an intervention for impingement syndrome of the shoulder joint. The conclusion of this review suggests that the best clinical outcome for improving subacromial impingement would occur with the implementation of scapular stabilization exercises either solely or as a part of shoulder rehabilitation. Heterogeneity of exercise interventions, coupled with outcome measures, prevented conclusions being drawn about the components of exercises that provides the best outcome.

Future studies aiming to evaluate the effect of scapular stabilization exercise in impingement syndrome in shoulder joint should aim to minimize potential bias by using larger samples, valid outcome measures, metrics related to scapular kinesis, homogenous subjects, flawless methodological design, and follow-up schemes to determine the sustainability of intervention effects. Further, if the future researches identify a significant positive effect for the scapular stabilization exercise for impingement syndrome, those studies should emphasize on the dose-response effect to guide clinical practice, for instance; intensity, frequency, sets, repetitions, interval or rest time, speed, and individualized or standard program.

\section{CONFLICT OF INTEREST}

No potential conflict of interest relevant to this article was reported.

\section{ACKNOWLEDGMENTS}

We sincerely thank the College of Medicine and Health Sciences, of the Mekelle University and University of Gondar for helping us with the internet service, online library access, and library services during the review process.

\section{REFERENCES}

Başkurt Z, Başkurt F, Gelecek N, Özkan MH. The effectiveness of scapular stabilization exercise in the patients with subacromial impingement syndrome. J Back Musculoskelet Rehabil 2011;24:173-179.

Bernhardsson S, Klintberg IH, Wendt GK. Evaluation of an exercise concept focusing on eccentric strength training of the rotator cuff for patients with subacromial impingement syndrome. Clin Rehabil 2011; 25:69-78.
Bury J, West M, Chamorro-Moriana G, Littlewood C. Effectiveness of scapula-focused approaches in patients with rotator cuff related shoulder pain: A systematic review and meta-analysis. Man Ther 2016;25: $35-42$.

Cochrane Effective Practice and Organisation of Care (EPOC). EPOC resources for review authors [Internet]. London: Cochrane, 2013 [cited 2020 Feb 2]. Available from: https://epoc.cochrane.org/resources/epoc-resources-review-authors.

Desmeules F, Côté CH, Frémont P. Therapeutic exercise and orthopedic manual therapy for impingement syndrome: a systematic review. Clin J Sport Med 2003;13:176-182.

Dewhurst A. An exploration of evidence-based exercises for shoulder impingement syndrome. Int Musculoskelet Med 2010;32;111-116.

Fatoye F. The economic impact of musculoskeletal pain. Pain Rehabil 2018;44:3-4.

GBD 2016 Disease and Injury Incidence and Prevalence Collaborators. Global, regional, and national incidence, prevalence, and years lived with disability for 328 diseases and injuries for 195 countries, 19902016: a systematic analysis for the Global Burden of Disease Study 2016. Lancet 2017;390:1211-1259.

Green S, Buchbinder R, Hetrick S. Physiotherapy interventions for shoulder pain. Cochrane Database Syst Rev 2003;(2):CD004258.

Greving K, Dorrestijn O, Winters JC, Groenhof F, van der Meer K, Stevens M, Diercks RL. Incidence, prevalence, and consultation rates of shoulder complaints in general practice. Scand J Rheumatol 2012;41:150-155.

Hanratty CE, McVeigh JG, Kerr DP, Basford JR, Finch MB, Pendleton A, $\mathrm{Sim} J$. The effectiveness of physiotherapy exercises in subacromial impingement syndrome: a systematic review and meta-analysis. Semin Arthritis Rheum 2012;42:297-316.

Hotta GH, Santos AL, McQuade KJ, de Oliveira AS. Scapular-focused exercise treatment protocol for shoulder impingement symptoms: threedimensional scapular kinematics analysis. Clin Biomech (Bristol, Avon) 2018 ;51:76-81.

Houglum PA. Rehabilitation of subacromial impingement starts at the scapula. J Orthop Trauma Rehabil 2013;17:54-60.

Kromer TO, Tautenhahn UG, de Bie RA, Staal JB, Bastiaenen CH. Effects of physiotherapy in patients with shoulder impingement syndrome: a systematic review of the literature. J Rehabil Med 2009;41:870-880.

Larsson R, Bernhardsson S, Nordeman L. Effects of eccentric exercise in patients with subacromial impingement syndrome: a systematic review and meta-analysis. BMC Musculoskelet Disord 2019;20:446.

Lloyd-Smith W. Evidence-based practice and occupational therapy. Br J Occup Ther 1997;60:474-478 .

Luime JJ, Koes BW, Hendriksen IJ, Burdorf A, Verhagen AP, Miedema HS, Verhaar JA. Prevalence and incidence of shoulder pain in the general 
population; a systematic review. Scand J Rheumatol 2004;33:73-81.

McQuade KJ, Borstad J, de Oliveira AS. Critical and theoretical perspective on scapular stabilization: what does it really mean, and are we on the right track? Phys Ther 2016;96:1162-1169.

Moezy A, Sepehrifar S, Solaymani Dodaran M. The effects of scapular stabilization based exercise therapy on pain, posture, flexibility and shoulder mobility in patients with shoulder impingement syndrome: a controlled randomized clinical trial. Med J Islam Repub Iran 2014; 28:87.

Moher D, Shamseer L, Clarke M, Ghersi D, Liberati A, Petticrew M, Shekelle P, Stewart LA; PRISMA-P Group. Preferred reporting items for systematic review and meta-analysis protocols (PRISMA-P) 2015 statement. Syst Rev 2015;4:1.

National Heart, Lung and Blood Institute (NHLBI). Study quality assessment tools. Bethesda (MD): NHLBI; 2014.

Oh LS, Wolf BR, Hall MP, Levy BA, Marx RG. Indications for rotator cuff repair: a systematic review. Clin Orthop Relat Res 2007;455:52-63.

Panagiotopoulos AC, Crowther IM. Scapular dyskinesia, the forgotten culprit of shoulder pain and how to rehabilitate. SICOT J 2019;5:29.

Schmitt L, Snyder-Mackler L. Role of scapular stabilizers in etiology and treatment of impingement syndrome. J Orthop Sports Phys Ther 1999; 29:31-38.

Seitz AL, McClure PW, Finucane S, Boardman ND 3rd, Michener LA. Mechanisms of rotator cuff tendinopathy: intrinsic, extrinsic, or both? Clin Biomech (Bristol, Avon) 2011;26:1-12.

Shankar P, Jayaprakasan P, Devi R. Effect of scapular stabilization exercise for type 2 scapular dyskinesis in subjects with shoulder impingement. Int J Physiother 2016;3:106-110.

Sterne JAC, Savović J, Page MJ, Elbers RG, Blencowe NS, Boutron I, Cates
CJ, Cheng HY, Corbett MS, Eldridge SM, Emberson JR, Hernán MA, Hopewell S, Hróbjartsson A, Junqueira DR, Jüni P, Kirkham JJ, Lasserson T, Li T, McAleenan A, Reeves BC, Shepperd S, Shrier I, Stewart LA, Tilling K, White IR, Whiting PF, Higgins JPT. RoB 2: a revised tool for assessing risk of bias in randomised trials. BMJ 2019;366:14898.

Struyf F, Nijs J, Baeyens JP, Mottram S, Meeusen R. Scapular positioning and movement in unimpaired shoulders, shoulder impingement syndrome, and glenohumeral instability. Scand J Med Sci Sports 2011;21: 352-358.

Struyf F, Nijs J, Mollekens S, Jeurissen I, Truijen S, Mottram S, Meeusen R. Scapular-focused treatment in patients with shoulder impingement syndrome: a randomized clinical trial. Clin Rheumatol 2013;32:73-85.

Takeno K, Glaviano NR, Norte GE, Ingersoll CD. Therapeutic interventions for scapular kinematics and disability in patients with subacromial impingement: a systematic review. J Athl Train 2019;54:283-295.

Turgut E, Duzgun I, Baltaci G. Effects of scapular stabilization exercise training on scapular kinematics, disability, and pain in subacromial impingement: a randomized controlled trial. Arch Phys Med Rehabil 2017;98:1915-1923.e3.

van der Windt DA, Burke DL, Babatunde O, Hattle M, McRobert C, Littlewood C, Wynne-Jones G, Chesterton L, van der Heijden GJMG, Winters JC, Rhon DI, Bennell K, Roddy E, Heneghan C, Beard D, Rees JL, Riley RD. Predictors of the effects of treatment for shoulder pain: protocol of an individual participant data meta-analysis. Diagn Progn Res 2019;3:15.

Virta L, Joranger P, Brox JI, Eriksson R. Costs of shoulder pain and resource use in primary health care: a cost-of-illness study in Sweden. BMC Musculoskelet Disord 2012;13:17. 